\title{
Super-large-scale flow visualization using natural snowfall for the study of utility-scale wind turbine flows
}

\author{
A. Abraham ${ }^{1,2}$, J. Hong ${ }^{1,2 *}$ \\ ${ }^{1}$ University of Minnesota, Department of Mechanical Engineering, Minneapolis, Minnesota, USA \\ ${ }^{2}$ University of Minnesota, St. Anthony Falls Laboratory, Minneapolis, Minnesota, USA \\ *jhong@umn.edu
}

With the rapid growth of wind turbine installation in recent decades, fundamental physical understanding of the flow around wind turbines and farms is becoming increasingly critical for further efficiency increases. However, the effort to develop this understanding is hindered by the significant challenges involved in modelling such a complex dynamic system with a wide range of relevant scales (blade boundary layer thickness at $\sim 1 \mathrm{~mm}$ to atmospheric scales at $\sim 1 \mathrm{~km}$ ). Additionally, conventional methods used to measure air flow around wind turbines in the field (e.g., lidar) are limited by low spatio-temporal resolutions.

To meet the need for a high-resolution field-scale atmospheric flow measurement technique, Hong et al. (2014) developed super-large-scale particle image velocimetry (SLPIV) using natural snowflakes as flow tracers. When illuminated at night and captured on video, the snowflakes provide sufficient signal for flow visualization and SLPIV in an area even above a scale of $100 \mathrm{~m}$. Moreover, due to the inertia of snow particles, they tend to be expelled from the centers of strong vortices, forming regions of low snow concentration, or voids, which become effective markers of coherent vortical structures in a turbulent flow field. When implemented in the near wake of a utility-scale wind turbine, the snow particle images are able to provide unprecedented visualization of all characteristic coherent flow structures (Fig. 1).
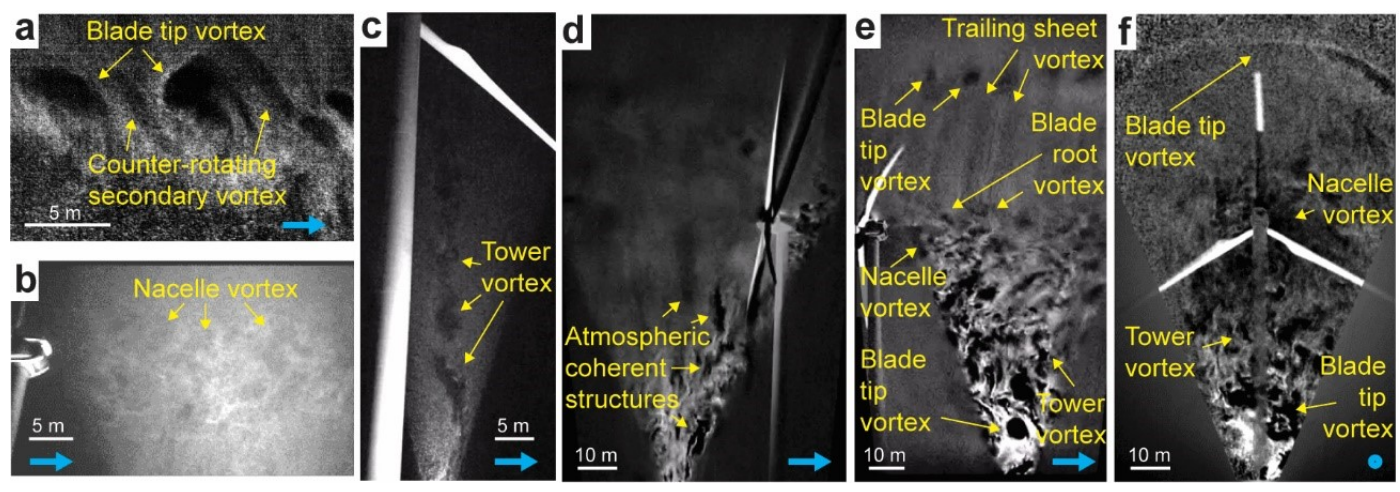

Figure 1: Sample images of snow particle patterns used for flow visualization and SLPIV measurements of (a) the bottom blade tip vortices, (b) turbine nacelle wake, (c) tower vortex tubes, (d) atmospheric flow approaching the turbine, (e) near-wake flow at the tower plane, and (f) near-wake flow at the plane normal to the flow direction.

For measurements in the plane parallel to the flow, quantitative flow information is extracted using cross-correlation between consecutive video frames, as in conventional PIV. For the relatively small field of view $(\sim 10 \mathrm{~m})$ measured in Hong et al. (2014), individual snow particles provide the signal for the crosscorrelation, enabling sufficient resolution to capture the velocity field around individual vortices shed from the turbine blade tips (Fig. 2a). For a large field of view on the order of $100 \mathrm{~m}$, the correlation used in SLPIV relies on the movement of large-scale patterns formed by the voids and clusters of snow particles, rather than individual snow particles, as described in detail by Dasari et al. (2019) and shown in Fig. 2(b). For measurements in the plane normal to the flow direction, additional image enhancement techniques are applied to snow particle images for robust extraction of the snow voids associated with helical blade tip vortices (Fig. 1f). The change of the near-wake blade tip vortices over time reveal the corresponding largescale wake movement in response to changes in atmospheric conditions and turbine operation, shown in Fig. 2(c) and described in detail in Abraham and Hong (2020). 

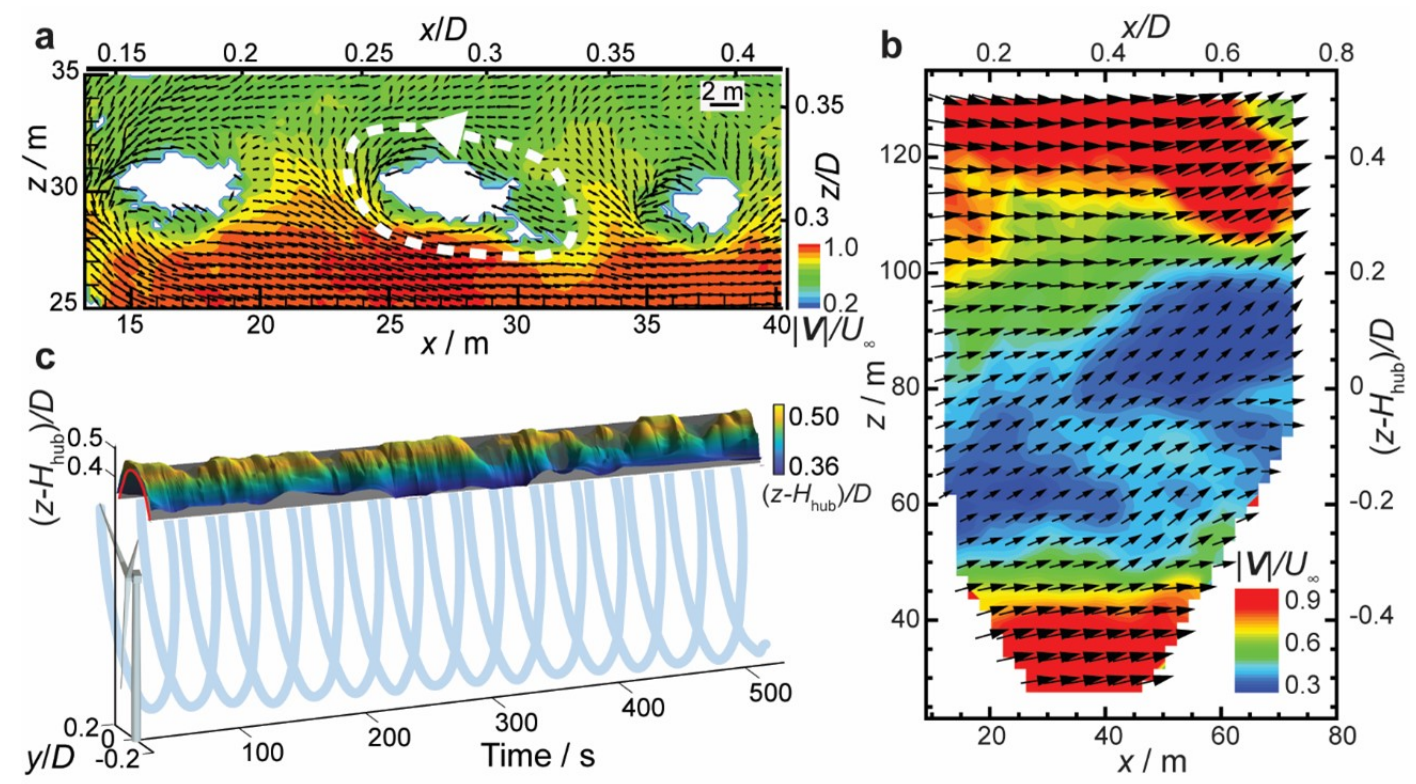

Figure 2: (a) A sample instantaneous velocity vector field around the bottom blade tip vortices superimposed with velocity magnitude contours. (b) A sample instantaneous velocity vector field with velocity magnitude contours (1:2 skip applied for clarity). (c) Sample time series of the top portion of the wake envelope extracted from snow particle images recorded in the plane normal to the flow direction.

These measurements have revealed several interesting behaviors of near-wake flows (e.g., wake contraction, dynamic wake modulation, enhanced momentum flux at the ground surface, etc.), and their connections with constantly-changing inflow and turbine operation, which are unique features of utility-scale turbines. These findings have demonstrated that near wake flows, though highly complex, can be predicted with substantial statistical confidence using information readily available from current utility-scale turbines. Such knowledge can be potentially incorporated into wake development models and turbine controllers for wind farm optimization in the future. Additionally, the SLPIV technique used for these measurements can be applied to the investigation of a wide variety of atmospheric flows. It has been implemented to analyze characteristics of large-scale boundary layer flow (Heisel et al. 2018) and the interactions between snowflake settling and atmospheric turbulence (Nemes et al. 2017). In the future, it can be further utilized to study other atmospheric flows, such as the wakes behind tall buildings and the flow through urban environments.

\section{References}

Abraham A and Hong J (2020) Dynamic wake modulation induced by utility-scale wind turbine operation. Applied Energy 257:114003

Dasari T, Wu Y, Liu Y, and Hong J (2019) Near-wake behaviour of a utility-scale wind turbine. Journal of Fluid Mechanics 859:204-246

Heisel M, Dasari T, Liu Y, Hong J, Coletti F, and Guala M (2018) The spatial structure of the logarithmic region in very-high-Reynolds-number rough wall turbulent boundary layers. Journal of Fluid Mechanics 857:704-747

Hong J, Toloui M, Chamorro LP, Guala M, Howard K, Riley S, Tucker J, and Sotiropoulos F (2014) Natural snowfall reveals large-scale flow structures in the wake of a $2.5-\mathrm{MW}$ wind turbine. Nature Communications 5:4216

Nemes A, Dasari T, Hong J, Guala M, and Coletti F (2017) Snowflakes in the atmospheric surface layer: observation of particle-turbulence dynamics. Journal of Fluid Mechanics 814:592-613 